\title{
A Construction of Online Luminance Analysis Service Website
}

\author{
Sung De Hong ${ }^{1}$, Jeong Tai Kim,** \\ ${ }^{1}$ Dept. of Interior Design, Shin Ansan University, 671 Choji-dong Ansan, 425-792, \\ Republic of Korea \\ 2, Dept. of Architectural Engineering, Kyung Hee University, 1732 Deogyeong-daero Yongin, \\ 446-701, Republic of Korea
}

Received 17 May 2015, Revised 16 November 2015, Accepted 10 December 2015

\begin{abstract}
In February 2012, Light Pollution Prevention Act was enacted and implemented in Korea. Under the new law, architectural exterior lightings and advertisement lightings will have to observe the permissible light emitting levels set by the enforcement ordinance of the law. In accordance with Article 12 of the law, the businesses which are legally required to observe the permissible light emitting levels should examine if their products satisfy legal requirements through the measurement of luminance. To measure the luminance of architectural external lightings and advertisement lightings, expensive measuring equipment is needed. For many small businesses, however, the measuring equipment is too expensive, and this kind cost burden may cause resistance against Light Pollution Prevention Act. In addition, the businesses are lack of expertise relating to the measurement of luminance. Therefore, this study proposed a concept of online luminance analysis services for the general public including the management and employees from the related industry. The online luminance analysis service adopted a method to analyze luminance by HDR-imaging videos using a common DSLR camera. In addition, a website to provide online services was constructed. The online luminance analysis services process proposed in this study include the followings: First, a website user should learn and follow how to capture and how to set the digital camera instructed on the website. Second, architectural exterior lightings and advertisement lightings should be captured using a digital camera. Third, the captured image files have to be uploaded on the website. Fourth, the uploaded image files should be analyzed by the website manager. Fifth, the analyzed results should be provided to the website users.
\end{abstract}

Keywords: Light Pollution, HDR Imaging, Luminance Measurement, Architectural Exterior Lighting, Advertisement Lighting.

\section{Introduction}

In February 2012, Light Pollution Prevention Act was enacted and implemented in Korea. Under the new law, architectural exterior lightings and advertisement lightings will have to observe the permissible light emitting levels set by the enforcement ordinance of the law. In accordance with Article 12 of the law, the businesses which are legally required to observe the permissible light emitting levels should examine if their products satisfy legal requirements through the measurement of luminance. To measure the luminance of architectural external lightings and advertisement lightings, expensive measuring equipment is required. For many small businesses, however, the measuring equipment is too expensive, and this kind of cost burden may cause resistance against Light Pollution Prevention Act. In addition, the businesses are lack of expertise relating to the measurement of luminance.

\footnotetext{
* Corresponding Author: Jeong Tai Kim, Tel.: +82-31-201-2539.

E-mail address: jtkim@khu.ac.kr
} 
To measure luminance value, using a digital camera is more economical than using a specialized luminance measuring device. Also, luminance analysis by using an image captured with a digital camera is regarded as an effective technology to measure non-uniform luminous surface, and it is reported to show low error percent. [1] In order to analyze luminance value of the images captured by digital camera online, the following conditions are required; first, it is necessary for the user to know how to set up a camera and capture with it, and second, it is necessary to provide the list of cameras which have tested calibration. Finally, it is necessary to build a system which can analyze the captured images. The purpose of the study is to consider technology related to analyzing luminance by using a digital camera (HDR imaging) and propose a website that provides an online luminance analysis service for ordinary users.

To build a website providing an online luminance analysis service, researches on the following contents has been conducted:

- First, principles to retrieve photometric information from the image captured by digital camera and the outline of the process (the principles of online luminance analysis service);

- Second, experiments to analyze luminance by using a HDR image and verification (the objects of advertisement lightings and architectural exterior lightings);

- $\quad$ Third, the provision of the concept to operate the website for online luminance analysis;

- $\quad$ Fourth, the organization of detailed contents for the website.

\section{Methods}

\subsection{Digital image and HDRI}

It is known that the human visual system can adapt to a dynamic range (DR) of up to 10,000:1. Meanwhile, because optical input and output devices such as an LCD monitor and digital camera have a fixed DR, they input and output expressible light within the range of the fixed DR. [2] In other words, if the scene's DR is high or the optical input and output device's DR is low, the device may not be able to record or reproduce the information of the brightness. Accordingly, a typical digital camera fails in recording or reproducing the details of an excessively bright or dark area in the actual scene. An HDR image is an image of which dynamic range is extended through the process of HDR imaging the limited DR image captured by a digital camera.

The LDR (Low Dynamic Range) image captured by a digital camera records the light information of a relatively narrower exposed range. The concept is that if the brightness information of the images recorded in this way is all combined, it is possible to record the light information that the actual scene has.

An HDR image is recorded in a RGBE format, and this records the radiance values of the actual scene as integer values. In order to express the range of light that human eyes can sense, each channel requires the bits in the range of the real number. And each channel should be recorded not as a fixed-point but as a floating-point value. The formula to convert the HDR image into the floating point RGB is as below: [3, 4]

$$
\text { red }=\frac{R}{255} * 2^{(E-128)}, \text { green }=\frac{G}{255} * 2^{(E-128)}, \text { b/ue }=\frac{B}{255} * 2^{(E-128)}
$$

The floating point RGB file is the information about color, and the process to convert it into tristimulus values that human can feel is required (CIE XYZ). Each pixel's CIE XYZ values come to be converted by applying the CIE 1931 2-degree XYZ color-matching functions from the floating point RGB value [4] (Figure 1).

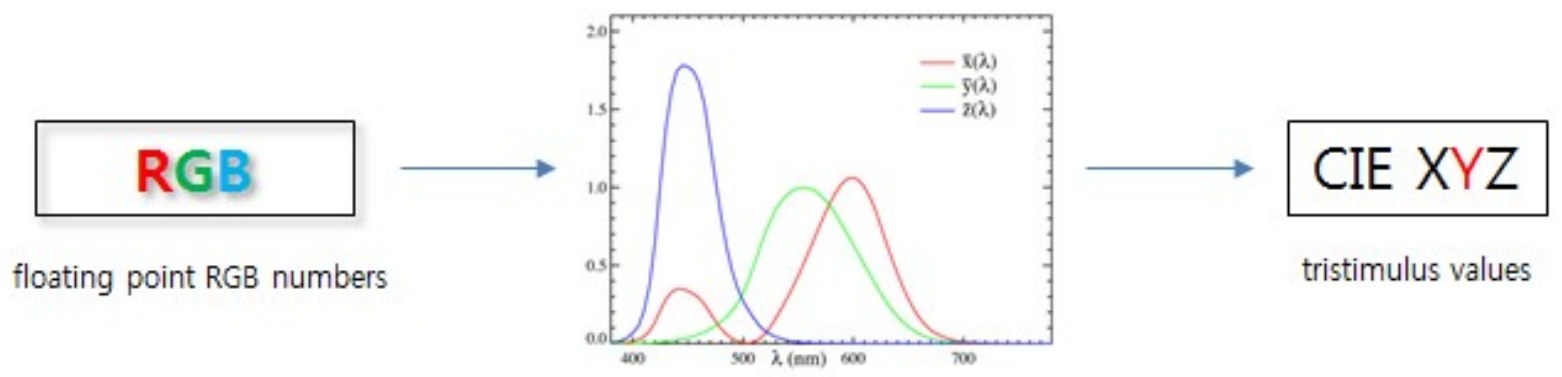

CIE 1931 Standard Colorimetric Observer Functions

Figure 1. HDR image analysis process 


\subsection{Luminance measurement using HDR technique}

In order to provide online luminance analysis service to measure luminance with HDR technique, comparison between the luminance value from HDR imaging and the luminance value measured with a photometer indicates the margin of error within $\pm 10 \%$. By conducting measurement on advertisement lightings (Case_1) and architectural exterior lightings (Case_2), which are the subjects regulated by the Light Pollution Prevention Act, the possibility is verified.

(1) Measurement set-up and equipment

As for the subjects of measurement, this study selected the area that had fewest factors to affect luminance in the process of measurement, had little traffic by humans or vehicles, and secured enough focusing distance between measuring subject and the measuring point (Table 1 ).

The time of measurement is from 22:00 to 24:00. As for the method of measurement, based on the 'Standard and Official Test Method', the point where the front of the object to be measured could be faced was chosen, and the angle between the device and the measuring subject was maintained as $45^{\circ}$ or less during that measurement.

The experiment was conducted in the following order: first, to comparatively analyze the results of luminance analysis using the HDR image and the value measured by the photometer and then to examine the error percent of each. It was Canon EOS 6D that was used to capture the LDR image to make the HDR image, and it was Photosphere that was used to create HDR image and analyse luminance.

Table 1. Measuring equipment and settings

\begin{tabular}{lc}
\hline Feature & Specification \\
\hline Digital camera & Canon EOS 6D \\
Lens & $24-105 \mathrm{~mm}$ \\
Image size & $5,472 \times 3,648$ \\
ISO & 100 \\
Image sensor & Full frame CMOS \\
White balance & Daylight / Fluorescent \\
\hline
\end{tabular}

As for capturing the LDR images, it started by capturing the real scene at the lower EV and then it was kept captured with $\pm 1 \mathrm{EV}$ increasing or decreasing at a time, finally increasing it to the higher EV. At this time, the aperture value was fixed, and only the shutter speed (TV) was varied (Figures 2 and 3).
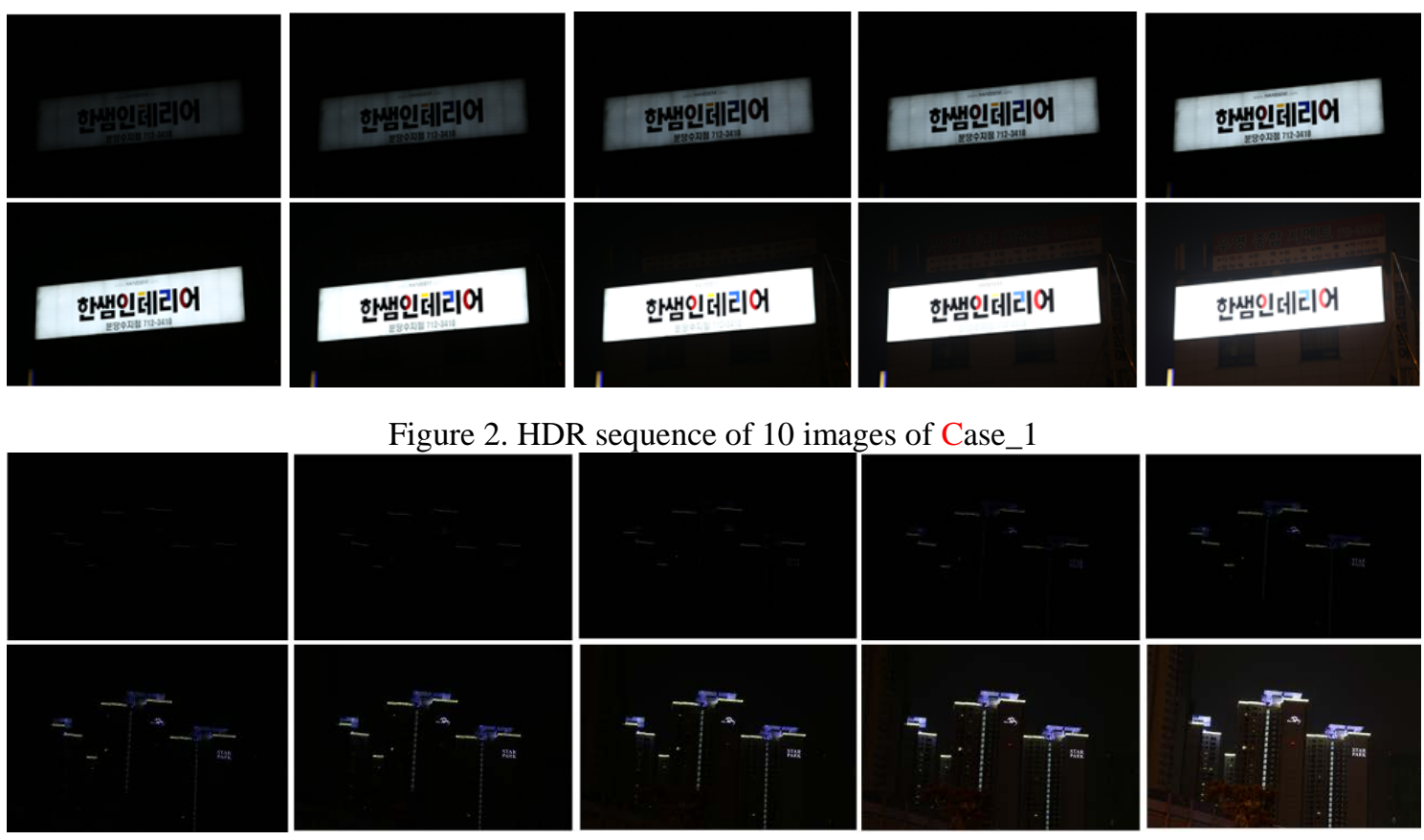

Figure 2. HDR sequence of 10 images of Case_1
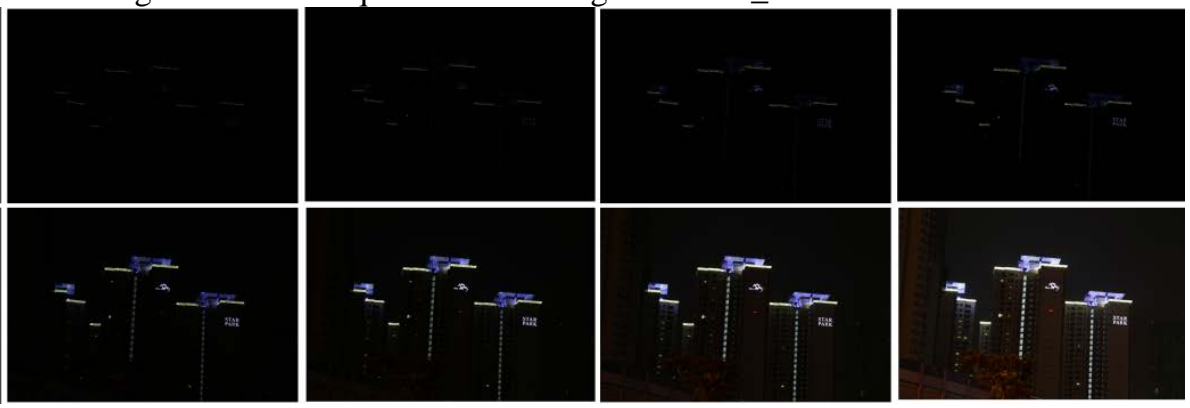

Figure 3. HDR sequence of 10 images of Case_2 
It was Minolta CA-2000 that was used to compare the luminance values obtained from the HDR image (Table 2). CA-2000 is an imaging photometer, so it is possible to check out the two-dimensional luminance distribution of a scene unlike spot luminance meter (LS-110).

Table 2. Specifications of CA-2000

\begin{tabular}{lc}
\hline Feature & Specification \\
\hline Measurement luminance range & 0.1 to $100,000 \mathrm{~cd} / \mathrm{m}^{2}$ \\
Lens & Telephoto lens \\
Accuracy(Luminance) & $\pm 3 \%$ \\
Accuracy(Chromaticity) & \pm 0.005 \\
Repeatability(Luminance) & $0.5 \%$ \\
Repeatability(Chromaticity) & 0.001 \\
\hline
\end{tabular}

(2) Results and analyses

According to the result of analysis on Case_1 and Case_2 HDR images with the photosphere program, the highest luminance of Case_1 is $526 \mathrm{~cd} / \mathrm{m}^{2}$, and the highest luminance of Case_2 is $895.78 \mathrm{~cd} / \mathrm{m}^{2}$.

In Case_1, the difference between the luminance value obtained from HDR imaging and that measured by using CA-2000 is $4.7 \mathrm{~cd} / \mathrm{m}^{2}$, and the error percent of measurement is found to be less than $1 \%$. And in Case_2, the difference of measured values is $83.215 \mathrm{~cd} / \mathrm{m}^{2}$, and the error percent of measurement is $8.5 \%$ (Figures 4 and 5 ).
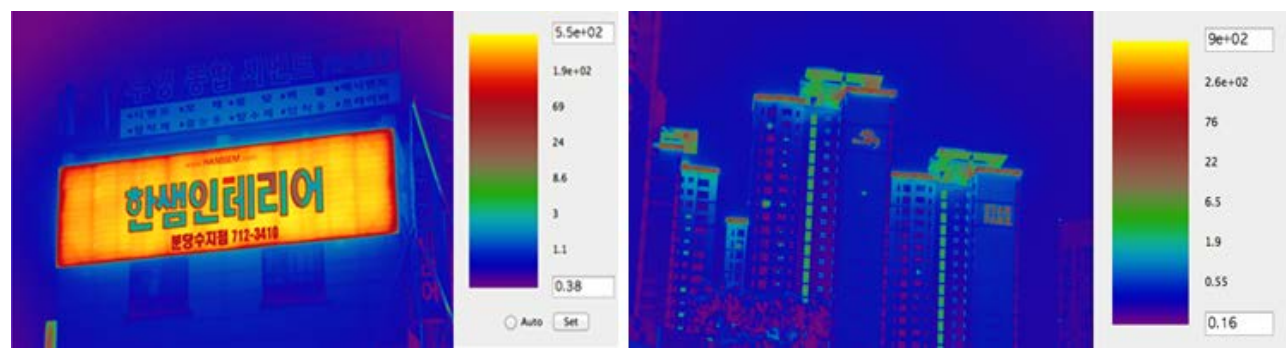

Figure 4. Luminance value obtained from HDR imaging
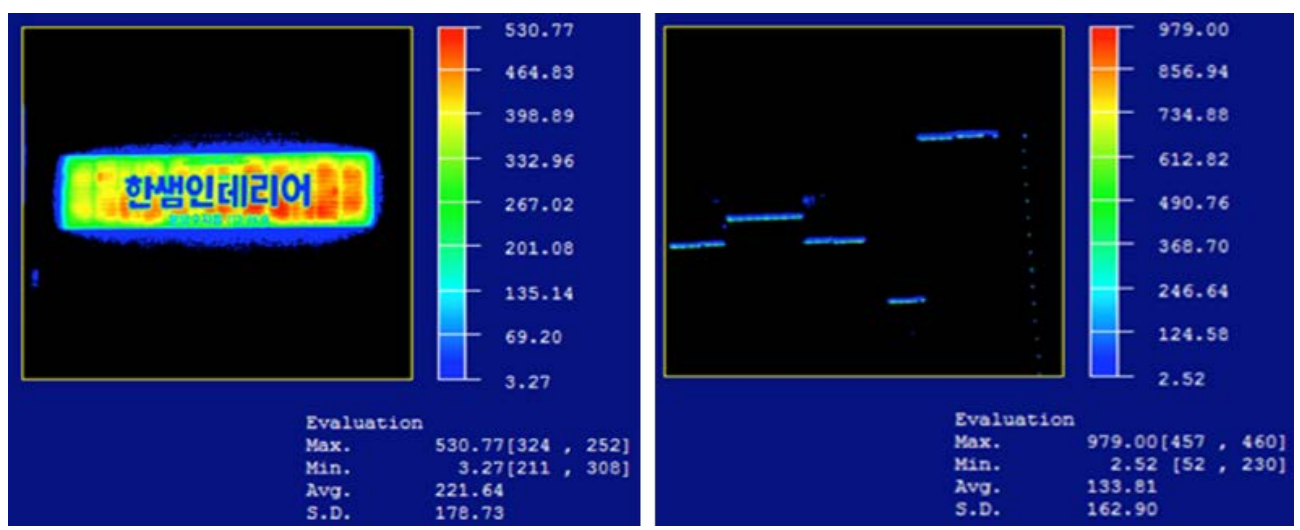

Figure 5. Luminance value using CA-2000

The luminance value obtained from HDR imaging and that measured by using CA-2000 are compared. In Case_1, the error percent is found to be relatively lower. And in Case_2, at the part where the bare lamp was exposed, the luminance values showed a noticeable difference, and at the part of indirect lighting, the error percent was low.

According to the result of comparing the luminance value obtained from HDR imaging with that measured by using CA-2000, the error percent was within the permissible error (10\%). Therefore, the result implies that online luminance analysis service can be provided for the image captured (LDRIs) by using a commercial digital camera (Table 3). 
Table 3. Results and error percent

\begin{tabular}{llc}
\hline Measurement & Case_1 (max.) & Case_2 (max.) \\
\hline HDR Imaging & $526 \mathrm{~cd} / \mathrm{m}^{2}$ & $895.78 \mathrm{~cd} / \mathrm{m}^{2}$ \\
CA-2000 & $530.77 \mathrm{~cd} / \mathrm{m}^{2}$ & $979 \mathrm{~cd} / \mathrm{m}^{2}$ \\
Error (\%) & $4.77 \mathrm{~cd} / \mathrm{m}^{2} \quad(0.9 \%)$ & $83.21 \mathrm{~cd} / \mathrm{m}^{2} \quad(8.5 \%)$
\end{tabular}

\section{Online luminance analysis service website}

\subsection{The concept of online luminance analysis}

Generally, a spot luminance meter is used to measure luminance. The spot luminance meter measures the surface of a subject seen through the lens and displays the measured value immediately. For Online Luminance Analysis Service, a digital camera that is widely used in everyday life is utilized. Unlike the method using a spot luminance meter, online luminance analysis service does not provide the measured value instantly. Therefore, the user captures the subject of light pollution with a digital camera and requests analysis on the captured file online and then receives the result of analysis according to the concept.

\subsection{The design and construction of the website}

The user who logs on to the website comes to know the digital camera models posted on the website and the method of capturing , and then captures either architectural exterior lightings or advertisement lightings. The user uploads detailed information about captured images and capturing in the Analysis Request Form. The uploaded image goes through a step of image data correction firstly by the website manager, and then, it goes to an HDR imaging step. After luminance analysis on the HDR image is completed, the maximum and average luminance values are calculated for architectural exterior lightings, and only the maximum luminance value is calculated for advertisement lightings to fill in a report (Figure 6).

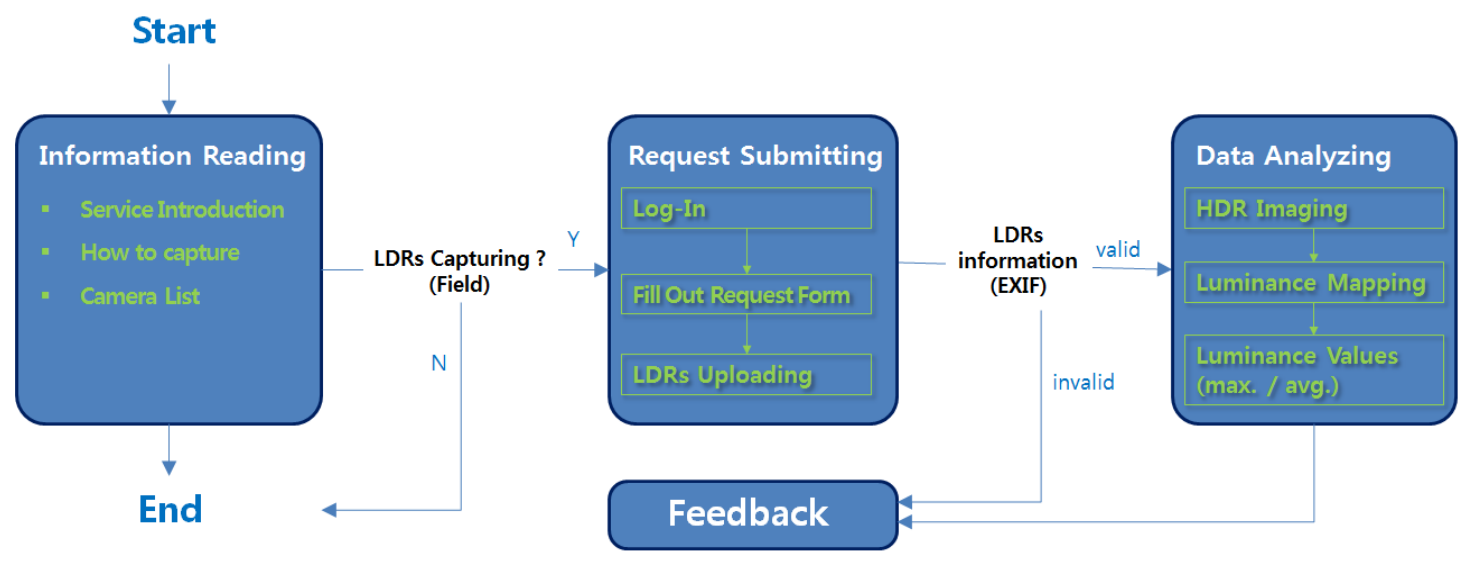

Figure 6. Website structure and online luminance analysis service procedure

The completed report comes to be uploaded on My Page of the user who requested the analysis. If the image uploaded by the user is partly corrected or it is not subject to the Light Pollution Prevention Act, a report recording 'Analysis Impossible' is delivered to the user.

\subsection{The design and construction of the website}

A new user to the website should acquire basic information about light pollution and understand knowledge about capturing. After that, he takes a picture of a subject causing light pollution and then fills out an analysis request form. Based on the website' s purpose, the website consists of the following four contents. On the left of the page, the content's titles are marked, and on the right, detailed contents are displayed. 
(1) Introduction to luminance analysis service

It is a page to present the background of the website opening to the users who log on to the website and inform them of the contents about the legal use of luminance analysis result. The idea that shorter wavelength visible light (450 to $500 \mathrm{~nm}$ ) is key in the treatment of SAD.

(2) Introduction to the Light Pollution Prevention Act

It posts the whole text of the Light Pollution Prevention Act being presently enforced.

(3) Introduction to the permissible light emitting levels.

It posts the permissible light emitting levels for architectural exterior lightings and advertisement lightings, which are the subjects of the website's luminance analysis service.

(4) Request for luminance analysis

The page for the request of luminance analysis is the main page on the website for online luminance analysis. It contains introduction to the principles to measure light emission, the list of digital cameras used for measurement, the method of capturing with a digital camera, and the fill-in and submission of an analysis request form.

\section{Discussion and conclusion}

Most subjects to be measured, which are advertisement lightings and architectural exterior lightings under the regulations of the Light Pollution Prevention Act, are made of high luminance light sources. And the light distribution is not uniform, and the image's DR is very high. The experiment for luminance analysis was conducted through HDR imaging in consideration of those characteristics of architectural exterior lightings and advertisement lightings, and the error percent was found to be less than $10 \%$. The results of the experiment prove that we can do luminance analysis of advertisement lightings and architectural exterior lightings by using a digital camera.

The website built as the result of this research targets ordinary people who lack of knowledge about luminance analysis. Luminance analysis is done by the website manager, and the users upload the images captured by a digital camera onto the website.

Therefore, the website contents are not about specialized information on luminance analysis but about information on light pollution and the method of capturing with a digital camera only. It means that it does not require website users to have either expensive measuring equipment or specialized knowledge to measure the luminance of architectural exterior lightings or advertisement lightings. With these findings, this study will be able to contribute to reducing the cost needed to measure luminance related with light pollution and also increasing national interest in light pollution.

\section{References}

[1]. Cai H. Camera aided luminance measurement of the luminous surfaces of different light sources. Proceedings of Architectural Engineering Conference (AEI) 2011: 272-279.

[2]. Jacobs A. High dynamic range imaging and its application in building research. Advances in building energy research. 2007; 1(1):177-202.

[3]. Inanici M. Evaluation of high dynamic range photography as a luminance data acquisition system. Lighting Research and Technology. 2006; 38(2):123-34.

[4]. Reinhard E, Heidrich W, Debevec P, Pattanaik S, Ward G, Myszkowski K. High dynamic range imaging: acquisition, display, and image-based lighting. Morgan Kaufmann; 2010. 